Results The rate of 'definite/probable' PID was highest among 20-24 year olds (410/100,000py; 95\% CI: 399-308). Between 2000 and 2011, the rate of 'probable/definite' cases among women declined in all age groups; on inclusion of 'possible' cases, rates of PID increased over the analysis period. Rates of EP (which were similar in HES and CPRD) were fairly stable overall between 1998 and 2010 (10.5/1,000 conceptions). EP rates increased with age and trends differed by age group, with decreases among women aged 30 years or older and small increases among $<30$ year olds.

Conclusions We observed homogeneity in declining rates of 'definite/probable' PID in all ages, but heterogeneity in EP trends by age. Interpreting trends in CT sequelae is complicated by diagnostic coding (PID), delays in sequelae onset (EP), variation in incidence by age and multiple aetiologies. EP trends in young women should become more informative in coming years for the evaluation of the impact of chlamydia screening. The likelihood of causes other than CT screening leading to changes in EP and PID need to be carefully assessed.

\section{P3.067 PREVALENCE OF CHLAMYDIA TRACHOMATIS AND NEISSERIA GONORRHOEAE IN HIGH SCHOOL STUDENTS BETWEEN 14 AND 19 YEARS-OLD USING A NON-INVASIVE TECHNIQUE}

doi:10.1136/sextrans-2013-051184.0527

'M Paredes, 'Y Gomez, ${ }^{2} \mathbf{M}$ Torres, 'M Fernandez, 'M Tovar. 'Universidad de La Sabana, Bogota, Colombia, ${ }^{2}$ STI Cochrane Review Group, Bogota, Colombia

Introduction Chlamydia trachomatis $(\mathrm{Ct})$ and Neisseria gonorrhoea $(\mathrm{Ng})$ are two of the most prevalent sexually transmitted infections (STI) in the world and affect mostly women younger of 25 years old causing sequels in their sexual health and fertility.

Objective to determine the prevalence of $\mathrm{Ct}$ and $\mathrm{Ng}$ of high school students in the centre area of the Cundinamarca, Colombia and associated factors for their infection.

Methods a cross-sectional study was developed. 1875 students between 14 and 19 years old of high school were identified in 11 small cities of a Colombian state. The selected students filled out a survey and gave a sample of urine for the detection of $\mathrm{Ct}$ and NG using real-time PCR. Before the survey, sexual health lectures were given to students, teachers and parents.

Results a sample of 972 sexually active students was obtained. The prevalence of Ct was 2.24\% (1.28-3.19 95\% CI) and $\mathrm{Ng} 0.09 \%$. ( $0 \%-0.28 \% 95 \%$ CI) No coinfection was detected. $21.4 \%$ of the girls with CT were asymptomatic (3/14). A association was identified between $\mathrm{Ct}$ and gender (chi2: 5.2, p:0.024), consume of psychoactive substances during the last sexual intercourse (chi2 4.4, p: 0.048) $y$ previous diagnosis of STI of their partner (chi2: 9.0; p: 0.006). $34.1 \%$ of the men and $79 \%$ of the women with $\mathrm{Ct}$ or $\mathrm{Ng}$ reported at least one symptom of STI in the last 3 months.

Conclusions This is the first study in Colombia using non-invasive techniques for the detection of Chlamydia and gonorrhoea. The prevalence of $\mathrm{Ct}$ and the frequency of asymptomatic cases were below compared with other studies. This can be explained because the area of the study is rural and some studies have shown differences between rural and urban areas. The population has a high frequency of symptoms which can be associated to other STIs and genital infections

\section{P3.068 PRIMARY SYPHILIS IS ON THE RISE IN GREECE: EPIDEMIOLOGICAL DATA FOR THE PERIOD 2005-2012 FROM A TERTIARY REFERRAL CENTRE IN ATHENS}

doi:10.1136/sextrans-2013-051184.0528

E Nicolaidou, A Kanelleas, C Stefanaki, I Stefanaki, G Bezrodnii, H Papadogeorgakis, A Katsambas, C Antoniou. Sexually Transmitted Infections Unit; 1st Dept. of Dermatology and Venereology, University of Athens, Athens, Greece
Background We conducted a retrospective study based on patients' records of the Sexually Transmitted Infections Unit of "A. Sygros" Hospital in Athens, Greece. This is a tertiary referral centre for sexually transmitted infections from an area that has almost half the population of Greece.

Methods We focused on Primary Syphilis (PS), having confirmed the diagnosis both clinically and serologically. We documented the total annual number of patients, the male/female ratio, and the patients' ethnic origin and sexual orientation.

Results We reviewed 1184 patients over the last eight years. The total number of patients with PS has risen from 111 in 2005 to 157 in 2012 , an increase of $41.44 \%$. The mean annual number is 144 . The mean male/female ratio is 4.85 , with a peak value of 8.50 in 2011. The majority of patients are of Greek origin, ranging from $67.4 \%$ to $87.2 \%$. Within the male patients group, it seems that the percentage of homosexuals has risen steadily from 2005 (20.7\%) up to 2011 (59.0\%) with a decline in 2012 (46.0\%). The mean value over eight years is $45.03 \%$.

Conclusion PS in Greece is on the rise. This is probably related to moving populations because of immigration and sex trade. Changes in sexual behaviour can also be a factor. However, the majority of patients are Greek, despite immigrant influx. Men are the most numerous subgroup among PS patients, representing more than $80 \%$ of the total number of patients. Furthermore, there seems to be a trend towards predominance of homosexual men as the core group among male patients.

\section{P3.069 ENHANCED OROPHARYNGEAL AND RECTAL TESTING FOR NEISSERIA GONORRHOEAE AND CHLAMYDIA TRACHOMATIS AT A PUBLIC STI CLINIC}

doi:10.1136/sextrans-2013-051184.0529

${ }^{1}$ H E L Reno, ${ }^{2} \mathrm{C}$ Brethauer, ${ }^{2} \mathrm{D}$ Spear, ${ }^{2} \mathrm{R}$ Knaup, ${ }^{1} \mathrm{~B}$ P Stoner. 'Washington University School of Medicine, Saint Louis, MO, United States; ${ }^{2}$ St. Louis County Department of Health, Saint Louis, MO, United States

Background Saint Louis, Missouri (USA) consistently reports high per capita rates of Neisseria gonorrhoeae (GC) and Chlamydia trachomatis (Ct). Asymptomatic testing for these STIs has traditionally involved genital testing alone. U.S. screening guidelines recommend GC/Ct testing at all sites of sexual exposure in men who have sex with men (MSM) and other high-risk groups. We instituted a policy to promote extragenital screening, in addition to genitourinary screening, for higher risk patients in a public health STI clinic. The purpose of this study is to assess implementation of this programme as well as the prevalence of STI at each site in our population.

Methods We conducted a retrospective study to compare rates of GC and Ct under enhanced testing conditions (genital plus rectal/ pharyngeal if exposed) from October 2012-January 2013. As part of the routine intake interview, patients presenting for testing were asked about sexual history and behavioural information.

Results Of 441 patients seen during the study period, $68.9 \%$ $(\mathrm{N}=304)$ were tested at an extragenital site. Among persons undergoing extragenital testing, $99.7 \%(\mathrm{~N}=303)$ had an oropharyngeal test, and $7.2 \%(\mathrm{~N}=22)$ had a rectal test performed. Extragenital testing showed that $4.6 \%$ of patients $(\mathrm{N}=14)$ had oropharyngeal GC, $1.0 \%(\mathrm{~N}=3)$ had oropharyngeal $\mathrm{Ct}$. Of the patients that underwent rectal testing, 9.1\% $(\mathrm{N}=2)$ had GC and $4.5 \%(\mathrm{~N}=1)$ had $\mathrm{Ct}$. Oral GC was found in $20.0 \%$ of patients with genital gonorrhoea $(\mathrm{N}=15)$. In addition, $12.0 \%$ of the positive tests were in patients that had negative genital site testing.

Conclusion Implementation of an enhanced GC/Ct testing policy identified significant numbers of patients with isolated oropharyngeal and rectal infection. The incidence of men with concurrent genital and oropharyngeal GC as well as the predominance of men accounting for the discordant oropharyngeal GC infection, may indicate the need for increased oropharyngeal testing in the general population. 